\title{
Integration of Multiple Segmentation Based Environment Models
}

\author{
SeungTaek Ryoo ${ }^{1}$ and CheungWoon $\mathrm{Jho}^{2}$ \\ 1 Department of Software, HanShin University, Korea \\ stryoo@hs.ac.kr \\ 2 Division of Digital Contents, DongSeo University, Korea \\ cwjho@dreamwiz.com
}

\begin{abstract}
An environment model that is constructed using a single image has the problem of a blurring effect caused by the fixed resolution, and the stretching effect of the 3D model caused when information that does not exist on the image occurs due to the occlusion. This paper introduces integration method using multiple images to resolve the above problem. This method can express parallex effect and expand the environment model to represent wide range of environment. 3
\end{abstract}

\section{Introduction}

The image-based modeling method is the one that is being studied the most due to its efficiency. This method enables real-time rendering because it extracts the geometric information from the image that represent the environment in the pre-processing stage. The objective of this paper is on real-time rendering for free navigation as realistically as possible. A method for acquiring the depthimage through image segmentation is suggested to construct the environment model. Also, the environment model has been made to be expandable through registration and integration of the multiple environment models.

The methods using the planes 1, 2,3 reconstruct the 3D model based the reference plane. Methods using the vanishing point and vanishing line 1,2 and the image editing method through interaction [3] are some examples of this method. The first method performs the modeling of the environment using the vanishing point based on the acquired plane, which makes it difficult to acquire an accurate environment model. The second method enables a more accurate modeling but requires user interaction using various tools. Horry 1 used a plane constructed using spider mesh to set the depth value and Criminisi 2 took into account the relationship between the plane parallel to the reference plane and the vanishing point in calculating the distance. Oh $[3$ used the floor parallel tool and the perpendicular tool to set the location on the reference plane. To resolve the

\footnotetext{
3 This work was supported by the Korea Research Foundation Grant(KRF-2004-003D00339).
} 
problem mentioned above, we used an environment modeling method based on depth image acquired through image segmentation 4. This method makes the environment modeling easier and can be implemented on an environment map.

\section{Multiple Environment Models}

A 3D model constructed using a single image has a problem of stretching effect when occluded object appear on the scene. A 3D environment model based on multiple images is required to resolve this problem. To do this, the integration method based on the corresponding line is suggested in this paper. the process of integrating the multiple environment models is as follows. First of all, the images acquired are reconstructed into 3D environment models using the method shown in previous chapter. Then the corresponding points are set from the image to be integrated and the 3D environment models are registered by using the transformation (translation, rotation, scaling) through the corresponding points. To acquire a more accurate environment model, the subdivision method is applied to integrate the environment models. Finally, the texel values are mixed and recreated to resolve the texture inconsistency effect to acquire a desired image.

\subsection{The Registration of the Environment Models}

Each image is divided into a floor, ceiling and surrounding objects 4 . The 3D environment models acquired through different viewpoints have the characteristic that the reference plane equation is equal to each other. By using this characteristic, the $3 \mathrm{D}$ coordinate registration can be simplified into a $2 \mathrm{D}$ coordinate registration. This means that the reconstructed environment models share the planes that form the floor and the ceiling which makes the registration of each environment model an easy task through 2D transformation. Figure 1 shows the process of integrating the 3D environment models using two corresponding point. Figure 1-a shows the setting of the two corresponding points from the image by user. The dotted line (corresponding line) indicates the vectors created by the first and second corresponding points. Figure 1-b shows the result image of the environment model $E_{a}$ created by the left image of Figure 1-a that has been translated by the environment model $E_{b}$ created by the right image. Figure 1-c shows the environment models created by rotating environment model $E_{a}$ around the first corresponding point. Figure 1-d shows the two environment models that have been created by scaling environment model $E_{a}$. We can see that the environment models can be registered by translating, rotating and scaling the models using only the two corresponding points.

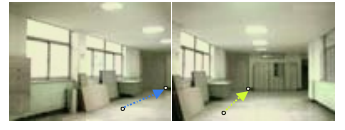

a. the corresponding point

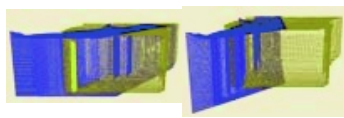

c. rotation

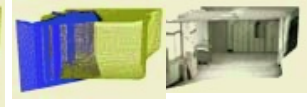

d. scaling

Fig. 1. the registration of the environment model 


\subsection{The Integration of the Environment Models}

we proposed the method of integrating the environment models after partition using a subdivision plane based on a corresponding line. Since two environment models based on only the two corresponding points have been registered, the environment models that are far away from the corresponding line have a larger displacement than those nearby. Therefore, it becomes difficult to discard the redundant areas through polygon comparison and analysis. We have integrated the two environment models using this feature between the corresponding line and the environment model. The environment model has been subdivided into two sub-models using the plane created based on the corresponding line and have chosen the sub-model with less redundant area to form an integrated environment model. Figure 2 shows the integration process of the environment model using the subdivision plane. Figure 2-a shows the process of selecting the area partitioned using the normal of the subdivision plane. Each dotted line indicates the intersection point between each subdivision plane and the environment model and the arrows indicate the direction of the normal of the plane. The sub-models are selected through this direction. Figure 2-b shows the environment models cut by each subdivision plane and Figure 2-c shows the environment model created by integrating the sub-models that have been partitioned. As shown in this figure, this method can be easily integrated the environment models to select and assemble the subdivided models. However, the model created during the integration of the two environment models has a problem of inconsistency in the connection area. To remove this seam, the texture samples of the two environment models that overlap each other must be blended for use. We have used the corresponding line for the consistency of the images in this paper. The seam can be fully removed by repeatedly applying texel blending.
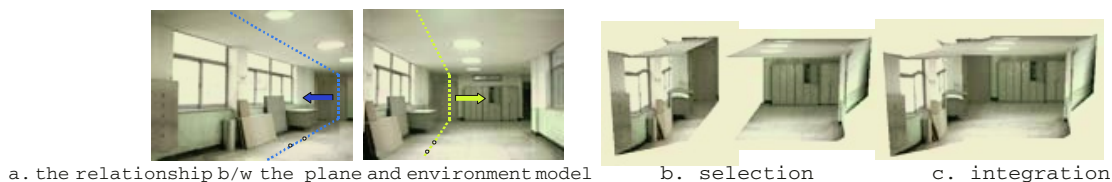

Fig. 2. the integration of the environment models using the subdivision plane

\section{The Results}

Figure 3 shows the process of constructing an environment model from three regular images acquired from different viewpoints and angle. In model1, the view orientation is towards the center of the hall, model 2 to the left-side, and model 3 to the right side to form the environment model. The two models, model 2 and 3 , are merged around model1. The merged models are divided into two sub-models using the subdivision plane, and the sub-model with less redundancy is selected for the integration. A seam is found on the connection area of an integrated 


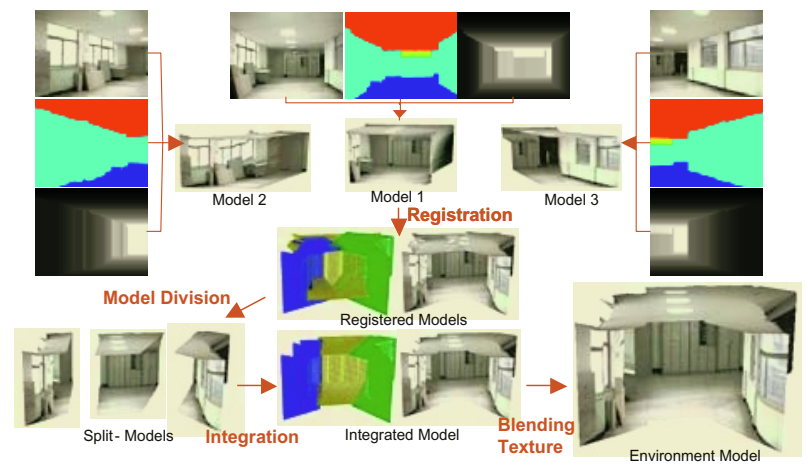

Fig. 3. the construction of the environment model using the multiple images

environment model. The environment model is reconstructed by blending the related texels from each texture map.

\section{Conclusion and Future Work}

The environment modeling method through the multiple images is able to create precise environment model that can freely change the viewpoint and has an optimal resolution image. It can also extend the navigation area through the integration of the environment model by adding a new environment image. The suggested environment modeling method using the multiple images has advantages as well as limitations which require further research. the 3D environment model acquired using the segmentation-based environment model is hard to merge and integrate precisely. Further studies will focus on a new way of acquiring a more precise 3D environment model without using the range image and also on ways for merging and integrating these new environment models automatically.

\section{References}

1. Y. Horry, K. Anjyo, K. Arai, "Tour Into the Picture: Using a Spidery Mesh Inter-face to Make Animation from a Single Image", SIGGRAPH '97, pp 225-232, 1997

2. A. Criminisi, I. Reid, A. Zisserman, "Single View Metrology", Int. J. of Computer Vision, v.40, n.2, pp. 123-148, 2000

3. Byong Mok Oh, Max Chen, Julie Dorsey, Fredo Durand, "Image-based modeling and photo editing", SIGGRAPH 2001: 433-442

4. SeungTaek Ryoo, "Segmentation Based Environment Modeling Using Single Image", ICIAR2004(LNCS3211), pp 98-105, 2004. 